\title{
LAser Shock Adhesion Test (LASAT) of Electron Beam Physical Vapor Deposited Thermal Barrier Coatings (EB-PVD TBCs)
}

\author{
G. Fabre ${ }^{1, a}$, V. Guipont ${ }^{1}$, M. Jeandin ${ }^{1}$, M. Boustie ${ }^{2}$, J.P. Cuq-Lelandais ${ }^{2}$, \\ L. Berthe ${ }^{3}$, A. Pasquet ${ }^{4}$, J-Y. Guedou ${ }^{4}$ \\ ${ }^{1}$ Centre des Matériaux, CNRS UMR 7633, MINES ParisTech, Competence Center for Spray \\ Processing, BP 87, 91003 Evry Cedex, France \\ ${ }^{2}$ Institut PPRIME, UPR CNRS 3346 (previously LCD), ENSMA, BP 109, 1 Av. Clément Ader, \\ 86961 Futuroscope, France \\ ${ }^{3}$ PIMM, UMR CNRS N8006, ARTS et METIERS ParisTech, 151 boulevard de l'Hôpital, 75013 \\ Paris, France \\ ${ }^{4}$ SNECMA, Villaroche, 77550 Moissy Cramayel, France \\ agregory.fabre@mines-paristech.fr
}

Key words: EB-PVD TBCs, Adhesion test, LASAT, Laser Shock, Spallation

\begin{abstract}
Damage prediction, adhesion strength and remaining lifetime of TBC are highly important data for understanding and preventing TBC spallation on blades. LAser Shock Adhesion Test (LASAT) is a powerful method to measure adhesion of coating due to its rapidity, simplicity and capabilities to distinguish different strength levels and the easy damage observation in case of TBCs. A new protocol of LASAT has been introduced in order to measure the adhesion level of the ceramic coating from the exploitation of the two-dimensional effects that promotes a shock wave pressure-dependent size of the damage. Finite element modeling, taking into account the TBCs dimensions, showed the edges effect on interfacial stress applied by laser shock.
\end{abstract}

\section{Introduction}

Temperature in combustor of gas turbine engines, both for aero-jet propulsion and land based industrial power generation, is limited by the melting point of the blade material. Single crystal superalloys with internal and external air cooling are the most advanced materials and designs for thermo-mechanical conditions in gas turbine engine. However, to improve power, thrust and NOx emissions, temperature in engines have to increase [1]. Last developments in this way are Thermal Barrier Coatings systems (TBCs) which allow superalloys surface temperature reduction up to $150^{\circ} \mathrm{C}$. TBCs are composed by four components, the superalloy, the bond coat (BC), the thermally grown oxide (TGO) and the $7 \mathrm{wt} \%$ yttria stabilized zirconia (7YSZ), each one having different functions.

TBCs failure has numerous origins [1] [2]. Impacts and abrasion by particles and foreign object can reduce the TBC thickness, reducing thermal protection or in some case can remove the ceramic top coat. Through a spallation process, another phenomenon affecting TBCs life expectancy and interfacial resistance to spalling is chemical segregation, in particular at the interface between the bond coat and the alumina layer [2]. Indeed this alumina layer is the critical area for spallation because of the energy concentration with effects of growth and thermal expansion misfit. Moreover, depending on the type of solicitations, i.e. isothermal mode or multiple thermal cycles, spallation occurs preferentially at $\mathrm{BC} / \mathrm{TGO}$ interface or TGO/7YSZ interface.

In the aeronautical industry, quality and performance of TBCs are controlled by high temperature thermal cycles. The limit number of cycles reached before complete spallation of the ceramic top layer is determined to measure the coating failure resistance. This test does not realize the measurement of the intrinsic mechanical adhesion but is an indirect assessment of it through the evaluation of the interfacial damages. As TBCs failure corresponds to cracks initiation, propagation 
and coalescence, several methods like interfacial indentation [3], barb pull-out methods [4], four point bending test [5] and direct pull tests [6] have been investigated. These methods are time expansive and need specific specimen shapes and appropriate fixture which do not allow a fast and easy-to-use industrial routine.

The LAser Shock Adhesion Test can be devoted to determine coating/substrate interface strength in case of ceramic/metal coating systems [7]. With LASAT (Fig. 1), a high power short pulse laser is focused (A) on a small material area. The sudden expansion of generated plasma (B) produces a high compressive stress pulse in the target. Compressive shock wave propagates (C) through material and is reflected when reaching the opposite free surface. The reverberation phenomenon changes the incident compressive shock wave in a tensile shock wave (D) which propagates to the opposite direction. In case of coatings, this tensile wave crosses the coating/substrate interface and can debond (E) the ceramic coating if the applied loading stress is superior to the intrinsic interface strength. Controlling the stress pulse level applied on the substrate by varying the laser energy densities, the tensile

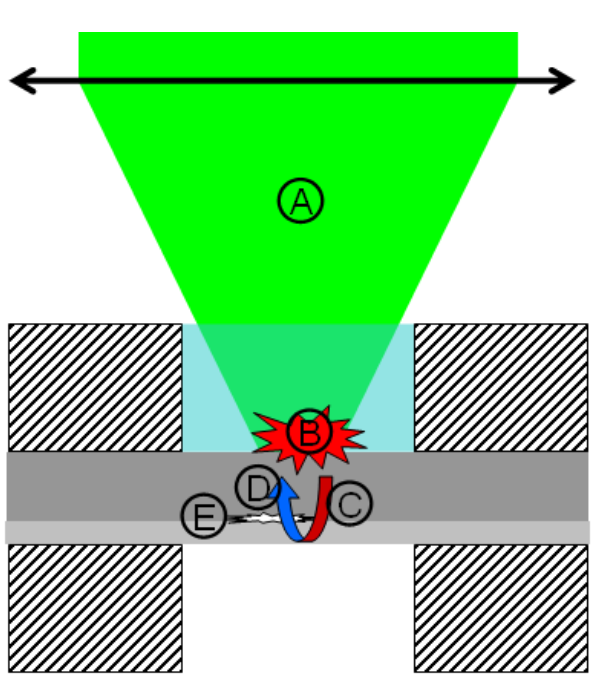

Fig. 1: LASAT principle stress generated at the interface can be varied. The adhesion level is determined through the lowest laser energy to debond the coating (LASAT threshold). LASAT is already applied to ceramic and metallic coatings by thermal spray technology both plasma and cold spray [7].

This paper introduces a new application of the LAser Shock Adhesion Test (LASAT) applied to TBCs obtained by EB-PVD. From the conventional LASAT with the detection of the so-called "LASAT threshold", an original approach to adhesion testing of ceramic coating is presented. This approach is based on the dimensional measurement of the interfacial damaged area and on the corresponding stress level calculation induced by laser shock. With this LASAT configuration, laser energy is selected to promote the ceramic coating debonding but with spallation (i.e. above the "LASAT threshold"). In the case of EBPVD TBCs, and thermal spray hydroxyapatite [7], LASAT induces a whitened spot in the ceramic, which is due to the presence of an interfacial crack. Therefore, in a first part devoted to experimental work, the relationship between the whitened spots diameters and the laser power density applied to generate the interfacial crack has been ascertained in the case of one typical industrial TBCs. In a second section, numerical modeling of LASAT on TBCs using Abaqus ${ }^{\circledR /} /$ explicit is implemented to simulate the stress time history involving the interfacial debonding. The last part of this paper introduces the description of a new and original approach of two-dimensional (2D) shock waves propagation that was necessary to ascertain the stress-dependent sizing of the interfacial crack both from FE calculations and experimental works.

\section{Materials and methods}

$\mathrm{Ni}$ based superalloy (AM1) plates with $(\mathrm{Ni}, \mathrm{Pt}) \mathrm{Al}$ bond coat were used as substrates $\left(50 \times 25 \times 1 \mathrm{~mm}^{3}\right)($ Fig. 2$)$. The ceramic coating (7YSZ) was $150 \mu \mathrm{m}$ in thickness. In this work, the presence of a thin alumina TGO interlayer $(\sim 1 \mu \mathrm{m})$ was also considered.

LAser Shock Adhesion Test has been generated by a Nd-YAG laser source. The Gaussian laser pulse $(532 \mathrm{~nm}, 7 \mathrm{~ns}, 1.5 \mathrm{~J}$ maximum) was focused on the superalloy side of the TBCs sample with a $2.5 \mathrm{~mm}$ spot diameter in water confined regime (Fig.1). Thickness and

\section{$1.8 \mathrm{GW} / \mathrm{cm}^{2}$}

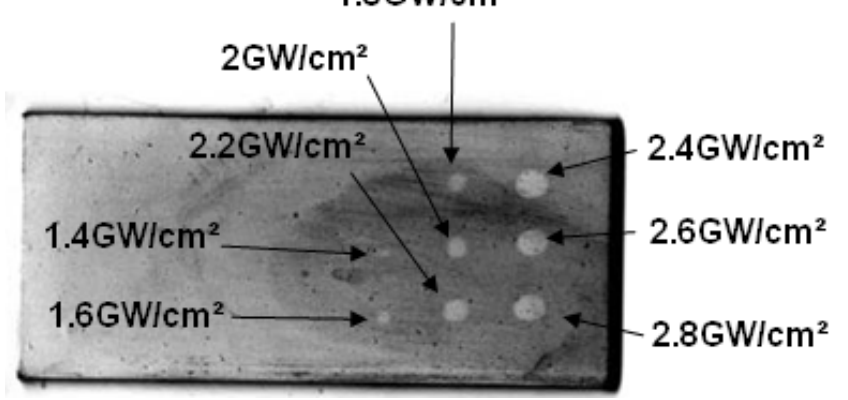

Fig. 2: Digital scan with modified contrast showing whitened spots on the top surface of TBCs after LASAT 
surface geometry of confining water are identical for each laser shock. Laser energy density has been varied to determine interfacial crack length dependence of LASAT. Indeed, observations of samples are performed by using a digital scan at 600 dpi for the whitened spots scanned from topview of the 7YSZ layer (Fig. 2). In addition, Scanning Electron Microscopy (LEO 1450 VP) observations of the cracked interface have been achieved on mounted cross-sections.

Shock wave propagation and temporal stress mapping of LASAT applied on TBCs has been modeled using Abaqus ${ }^{\circledR} /$ Explicit 6.8. An axisymmetrical model was developed since the surface of laser spot was circular. The material data used were found in the literature.

\section{Results and Discussions}

Experimental results. LASAT applied to TBCs can induce a whiteness change as obviously shown in Fig. 2 which is a modified contrast view on top surface of a shocked specimen. The color modification can be seen by visual inspection but, better evidence with less discrepancy was given using a digital scanning device with enhanced contrast. SEM cross section observations showed a clear relationship between whitened spot location and debonding crack at the interface, i.e. near the thin alumina layer. It was ascertained that each whitened spots corresponded to failure detection by SEM cross section and vice versa. Fig. 3 exhibits the interfacial crack induced by LASAT. The failure occurred at 7YSZ/alumina interface, or at the alumina/bond coat interface or also could be located through the alumina layer.
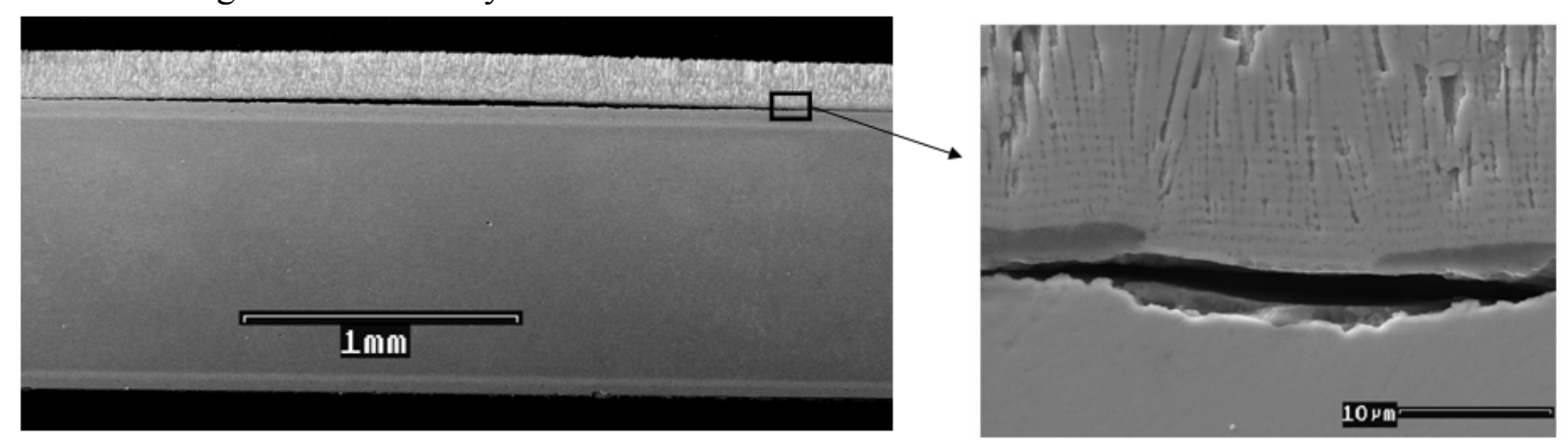

Fig. 3: Interfacial failure in TBCs due to LASAT

Damage induced by LASAT has a great interest because of the same localization with typical failures generated by spallation upon high temperature cyclic oxidation [1][2]. Experimentally, on a same coated TBC sample, there were different types of damages depending on laser intensity. First, laser energy and corresponding stress pulse was under the debonding threshold. Then, with increasing laser intensity (above the LASAT threshold), the shock wave induced one interfacial crack, but the coating remained attached on the rest of the ceramic, leading to a humped region. In this particular case, a change in whiteness of the 7YSZ is observed. For very high laser intensities, coating ejection has been observed (a ceramic chip is created and removed from the substrate).

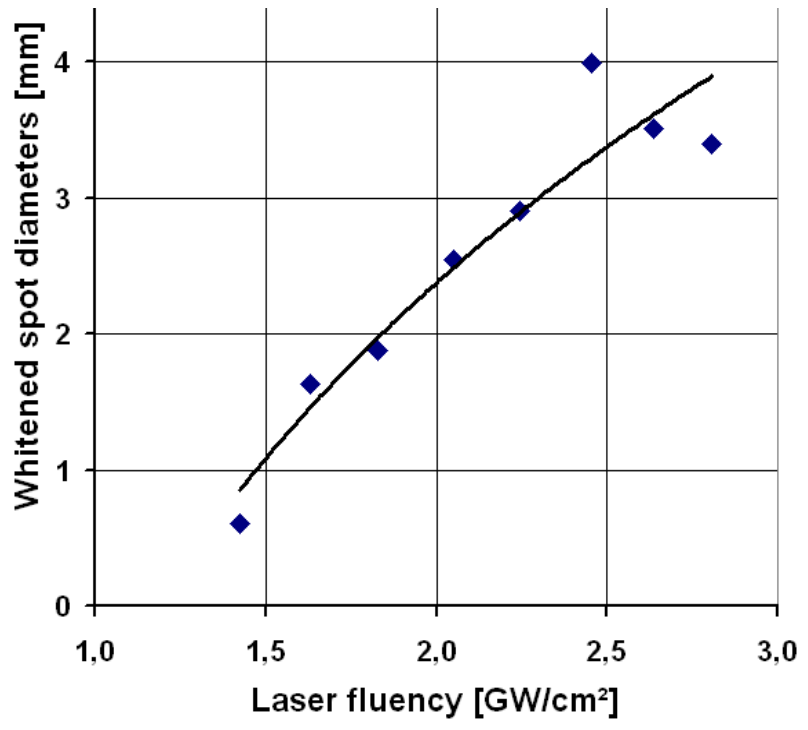

Fig.4: White spot diameter vs. Laser fluency plot for a LASATed TBCs

(Ølaser $=2.5 \mathrm{~mm})$ 
As it is shown in Fig. 4, the whitened spot size increases with increasing laser intensity. Indeed, the white spot diameter versus the laser intensity evidences a logarithmic evolution of experimental data. This statement has been at the origin of the new LASAT protocol devoted to TBC from the sizing of the whitened spot diameter to determine the adhesion level.

Modeling. The shock wave propagation through the multilayered TBCs has been modeled to show the stress time history involved by laser shock as mentioned by Wang et Al [8]. Axisymmetrical calculations have been performed with Abaqus ${ }^{\circledR} /$ explicit to simulate shock wave propagation and stress repartition along interface. The laser energy deposition is assimilated to an equivalent pressure loading with a $1.25 \mathrm{~mm}$ radial top-hat repartition. The pressure loading time repartition is supposed to be a 7ns FWHM Gaussian shape. The 7YSZ layer has been considered as a $150 \mu \mathrm{m}$ thick homogeneous material. The TGO was $3 \mu \mathrm{m}$ thick. The Bond Coat and superalloy were modeled by a single layer with $900 \mu \mathrm{m}$ in thickness. Contacts between meshed layers are perfect (Tie constraint). The crack propagation is only governed by the failure criteria defined in the TGO layer. Under intense shock pressure, material dynamic behavior can be described by the Johnson-Cook law without temperature-dependent softening component where $\varepsilon_{P}$ represents the plastic strain and $\dot{\varepsilon}$ is the strain rate:

$$
\sigma=\left[A+B \cdot \varepsilon_{P}{ }^{n}\right] \cdot\left[1+C \cdot \ln \left(\frac{\dot{\varepsilon}}{\varepsilon_{0}}\right)\right]
$$

Dynamic behavior of TBCs has not been investigated in the past. Since to the long process to determine law parameters for superalloy and each layer, coefficients $\left(A, B, C, \varepsilon_{0}\right.$ and $\left.n\right)$ of Al, a well known material under shock, have been used. Moreover, the Hugoniot is described by a linear relationship $D=C o+S \cdot u$, where $D$ is the shock velocity and $u$ the particle velocity. Finally, density $\rho$, Shear modulus $G(G=E /[2 \cdot(1+v)]$ with $E$ Young's modulus and $v$ Poisson's ratio) and the Grüneisen parameter $\Gamma$ for the Mie-Grünesein equation of state are needed for calculations. Parameters used for each material are detailed in Table 1.

Table 1: Parameters used for materials in simulations

\begin{tabular}{|c|c|c|c|c|c|c|c|c|c|c|c|}
\hline & \multirow{2}{*}{$\begin{array}{l}\text { Density } \\
p\left[\mathrm{~g} / \mathrm{cm}^{3}\right]\end{array}$} & \multirow{2}{*}{$\begin{array}{c}\text { Shear Modulus } \\
\mathrm{G}\left[10^{11} \mathrm{~Pa}\right]\end{array}$} & \multirow{2}{*}{$\begin{array}{c}\text { failure criteria } \\
\text { Cut off }\left[10^{11} \mathrm{~Pa}\right]\end{array}$} & \multicolumn{3}{|c|}{ EoS } & \multicolumn{5}{|c|}{ Johnson-cook } \\
\hline & & & & $\mathrm{CO}[\mathrm{cm} / \mathrm{s}]$ & 5 & $\Gamma$ & $\mathrm{A}\left[10^{11} \mathrm{~Pa}\right]$ & $\mathrm{B}\left[1 \mathrm{O}^{11} \mathrm{~Pa}\right]$ & $\mathrm{c}$ & $\mathrm{n}\left[10^{11} \mathrm{~Pa}\right]$ & $\varepsilon_{0}\left[\mu s^{-1}\right]$ \\
\hline AM1 $900 \mu \mathrm{m}$ & 8,67 & 0,5 & none & 0,4119 & 1,806 & 1,88 & 0,0013 & 0,01042 & 0,015 & 0,4 & \\
\hline Zirconia $150 \mu \mathrm{m}$ & 6,6 & 0,78 & none & 0,7705 & 1,28 & 2 & & & & & \\
\hline Alumina $3 \mu \mathrm{m}$ & 3,96 & 154 & 0,00076 & 0,7705 & 1,28 & 2 & & & & & \\
\hline
\end{tabular}

Two-dimensional stress waves are given in Fig.5. The edge effects of shock wave, lateral and non-straight propagation are exhibited and were already described by Boustie et al. [9-10]. Upon high shock, a principal shock wave (A region), one-dimensional, is induced on the normal direction of the surface. From the rim of the laser spot, two-dimensional shock waves propagate spherically both on the laser shock central axis and in the lateral direction. One part, which propagates towards the laser shock axis is a tensile shock wave. It follows the main shock wave. The second part is compressive shock wave which propagates forward the radial/lateral direction of the laser-irradiated area (B region). 
By reflection on the free surface (YSZ/air), Fig. 5.b, 1D tension wave propagates in the opposite direction, crossing through the $3 \mu \mathrm{m}$ thick alumina. In Fig. 5.b, 2D effects on the stress distribution along the radial axis after the shock wave reflection are described by the color map. The Figure 5.c exhibits the stress distribution along the interface from shock axis in the radial direction. The planar 1D wave induces a constant stress level only near the shock axis (A region) whereas a stress decreasing ( $\mathrm{B}$ region) due to lateral edge effects is observed. From this LASAT FE model, in one hand a modification of the laser spot diameter will affect the stress distribution along the TGO. Thus, for a large laser spot diameter regarding to the TBCs thickness, the $\mathrm{B}$ region which represents $2 \mathrm{D}$ effects of shock wave could be neglected. In this case the 1D shock wave assumption is assumed and the presence of an interfacial crack can be modeled [8]. In the other hand, when the laser spot is small compared to the TBCs thickness, the edge effects (region B) will be emphasized leading to a stress gradient along the interface. Through this crucial approach to laser spot size, it is claimed that the interface could be tested with different levels of tensile stresses.

\section{Conclusion}

Using LAser Shock Adhesion Test, TBCs has been characterized. The tensile wave can induce, depending on applied laser intensity, an interfacial failure between Bond Coat and Top Coat. For TBCs, the failure is directly observed by a whitened spot on coating side of specimens. It has been demonstrated that the whitened spots and the corresponding cracks have same geometries. Therefore, the whitened spots have been dimensioned to directly determine the crack sizes without SEM micrographs. It has been evidenced the dependencies between the increasing whitened spots size when increasing the laser fluency. This result expands the conventional LASAT which only consisted in determining the laser intensity threshold to create an interfacial crack. A new protocol has been introduced in order to measure the adhesion level of the ceramic coating from the exploitation of the two-dimensional effects of shock waves. With modeling, the spatial stress distribution in TBCs depending to the laser spot diameter was described. A tensile stress gradient along the interface was simulated. Works are in progress in order to understand 2D shock waves and crack propagation upon laser shock. 


\section{References}

[1] A. Feuerstein, J. Knapp, T. Taylor, A. Ashary, A. Bolcavage and N. Hitchman: Technical and Economical Aspects of Current Thermal Barrier Coating Systems for Gas Turbine Engines by Thermal Spray and EBPVD: A Review. Journal of Thermal Spray Technology, Vol. 17 (2007), p.199

[2] A.G. Evans, D.R. Mumm, J.W. Hutchinson, G.H. Meier and F.S. Pettit: Mechanisms controlling the durability of thermal barrier coatings. Progress in Materials Science, 46 (2000), p. 505

[3] J. Sniezewski, V. Vidal, P. Lours and Y. LE Moult: Thermal Barrier Coatings adherence and spallation: Interfacial indentation resistance and cyclic oxidation behaviour under thermal gradient. Surface \& Coatings Technology, 204 (2009), p. 807

[4] S.Q. Guo, D.R. Mumm, A.M. Karlsson and Y. Kagawa: Measurements of interfacial shear mechanical properties in thermal barrier coating systems by a barb pullout method. Scripta Materiala, 53 (2005), p.1043

[5] H.A. Bahr, H. Balke, T. Fett, I. Hofinger, G. Kirchhoff, D. Munz, A. Neubrand, A.S. Semenov, H.J. Weiss and Y.Y. Yang: Cracks in functionally graded materials. Materials Science \& Engineering, A362 (2002), p.2

[6] M. Gell, E. Jordan, K. Vaidyanathan, K. McCarron, B. Barber, Y.H. Sohn and V.K. Tolpygo: Bond strength, bond stress and spallation mechanisms of thermal barrier coatings, Surface \& Coating Technology, 120-121 (1999), p. 53

[7] V. Guipont, M. Jeandin, S. Bansard, K.A. Khor, M. Nivard, L. Berthe, J.P. Cuq-Lelandais and M. Boustie: Bond Strength determination hydroxyapatite coatings on Ti-6Al-4V substrates using Laser Shock Adhesion Test (LASAT). Submitted to Journal of Biomedical Materials Research (2010)

[8] Y. Liang, X. Bi and J. Wang: Numerical simulation of laser induced thin film delamination. Thin Solid Films, 516 (2008), p.971

[9] M. Boustie, J.P. Cuq-Lelandais, C. Bolis, L. Berthe, S. Barradas, M. Arrigoni, T. De Resseguier and M. Jeandin: Study of damage phenomena induced by edge effects into materials under laser driven shocks. J. Phys D.: Applied Physics, 40 (2007), p.7103

[10] M. Boustie, J.P. Cuq-Lelandais, L. Berthe, C. Bolis, S. Barradas, M. Arrigoni, T. De Resseguier and M. Jeandin: Damaging of materials by bi-dimensional dynamic effects. Shock Compression of Condensed Matter, Vol. 955 (2007), p.1323 